\title{
Facing the challenge of COVID-19 pandemic in Bangladesh: Critical Care Physicians need to define their role and share the burden
}

\author{
Mohammad Omar Faruq
}

Since the first cases of corona virus disease 2019 (COVID-19), caused by severe acute respiratory syndrome coronavirus 2 (SARS-CoV-2), were identified in China in December, 2019, Bangladesh began to pick up COVID positive cases beginning March 2020. Initially there was paucity of testing kits for RT PCR (Reverse Transcription Polymerase Chain Reaction) and initially there was only one testing lab for COVID 19. So primarily only people who had travel history and travelers arriving at the airport were tested. Institute of Epidemiology, Disease Control and Research of Bangladesh (sole govt. centre for epidemiological studies) otherwise known as IEDCR, picked up first 3 positive cases on March $082020^{1}$. Initially the number of positive cases were surprisingly low compared to our potentially exposed population. Since April 04 Bangladesh witnessed gradual increase in positive cases as number of testing centres were established in different parts the country and our country received plenty of testing kits from abroad. According to IEDCR, up till April 16 2020, 17000 tests were done and our country witnessed 1572 total positive cases and total number of COVID positive deaths added up to sixty. Since then number of positive cases and number of COVID related deaths have been slowly and steadily rising at an arithmetic progression.

Unlike South Korea and Switzerland where mass testing has been done, Bangladesh does not have the logistic capacity to screen majority of its population. It could only test people with symptoms, history of contact with positive cases or with symptoms and with history of foreign travel.

According to Claire Mills ${ }^{2}$ approximately 15-20 per cent of patients with COVID-19 require hospitalization and six per cent require intensive care for a duration of between 3 and 6 weeks. This pandemic is likely to lead to the disruption of basic medical services and emergency facilities, the de-prioritization of treatment for other life-threatening diseases, conditions and for other chronic infectious diseases everywhere. Such scenario has significant negative impact on country like Bangladesh where the health system is already fragile.

Vincent et al. ${ }^{3}$ described three scenarios while interpreting death from COVID -19. The first scenario involves ICU patients dying despite full intensive care support including mechanical ventilation. The second scenario involves death with limitation of life sustaining therapies along with old age, co morbidities or because of lack of personnel, beds, or materials or combination of both circumstances. The third scenario relates to patients coming from high COVID infection area admitted into ICU or hospital whose deaths are not related to COVID- 19 but admitted for sepsis, trauma, kidney disease, malignancy etc. and found COVID positive during hospital stay and eventually die from initial conditions. Bangladesh somehow fits mostly into second scenario and its resource constrain status has been an important issue.

In case of COVID pandemic time to respond is very short, and in case of mass infection it is unlikely that human and infrastructure resources can be mobilized or indeed created at scale quickly enough to ramp up intensive care to clinically effective levels. Emphasis should be placed on essential emergency and critical care where appropriate for those patients in low resource setting who do become critically unwell. Basic interventions such as delivering oxygen to patients, lying on their front ("proning"), suction, chest physiotherapy, and appropriate use of antibiotics for bacterial infections will help improve outcomes. Decision makers should focus on scaling-up resourcing and capacity development for ward-level care for the much larger proportion of patients that are likely to need $\mathrm{it}^{4}$.

When resource constrain becomes acute, one recently published artcle ${ }^{5}$ gives us a guideline about initial approach to critically ill patients with suspected COVID-19. For low threshold for suspecting COVID-19 consider full PPE for ICU staff. Consider critical care triage to ration scarce resources in pandemic and prioritize patients who will benefit from ICU care. Admit to single airborne infection isolation room with negative pressure in ICU. If negative-pressure room unavailable, admit to normal-pressure single room in ICU. If single room unavailable, cohort similar cases in shared room with beds spaced apart in ICU. If ICU unavailable, consider surge beds outside of ICU. If surge beds unavailable, consider transfer to designated hospitals and ICUs. Collect respiratory tract samples for RT-PCR, preferably sputum or endotracheal aspirates. If negative, repeat as appropriate based on index of suspicion.

So how do we define critical care in a resource poor setting? It is defined in a resource poor or constrained setting by the provision of care for life-threatening illness without regard to the location, including the pre-hospital, emergency, hospital wards, and intensive care setting. Such definition also emphasizes that in order to provide quality critical care, at any capability level, resource limited countries or health-care bodies should strengthen their primary care, basic emergency care, and public health systems. It is also suggested that critical care providers use protocols to combine workable approaches that are also cost effective and efficient. Resource-poor settings offer a unique challenge to the provision of mass critical care to vulnerable victims ${ }^{6}$. 
There are some lessons a country with poor resource setting like Bangladesh should learn from China as how to handle management and prevention of COVID epidemic ${ }^{7}$. They are: a) Provide clear guidance about the degree and scope of lockdowns. b) Track implementation of lock down to individuals, apartments, houses, communities, organizations, public facilities and city management. c) Keep essentials like food and supplies flowing through organized, government-controlled systems. d) Designate infectious disease care and management facilities to isolate, monitor and treat positive cases. e) Establish electronic recording and tracking systems and local response teams to handle identified cases 24/7. f) Establish centralized reporting and communication channels to keep citizens informed. It is heartening to know that ministry of health govt. of Bangladesh has been paying attention to these interventions and has begun implementing them in order of priority.

There is an important preventive issue relevant to critical care physicians and nurses. It is about exposure of our health care workers to COVID-19 infection. If we had to quarantine every health-care worker who might have come into contact with a COVID-19 patient, we would soon have no health-care workers left. What happens when someone unexpectedly tests positive - say, a hospital health care worker or a patient in a primary-care office or an emergency room or in ICU? Bangladesh should follow what Singapore or Hong Kong practises $^{8}$. In Hong Kong and Singapore, they don't shut the place down or put everyone under home quarantine. They do their best to trace every contact and then quarantine only those who had close contact with the infected person. In Hong Kong, "close contact" means fifteen minutes at a distance of less than six feet and without the use of a surgical mask; in Singapore, thirty minutes. If the exposure is shorter than the prescribed limit but within six feet for more than two minutes, workers can stay on the job if they wear a surgical mask and have twice-daily temperature checks. People who have had brief, incidental contact are just asked to monitor themselves for symptoms.

Now we turn our attention to the demography of Bangladesh in order to assess the potential of severity of this pandemic. Our country currently has a population of 164 million with a population density of 1265 per Sq. KM ${ }^{9}$. According to 2018 World Bank data, per capita annual GDP was 1750 US \$ (BDT $410 /-$ per day) ${ }^{10}$. We have to find out why the bite of COVID pandemic is so minimal in such densely populated and impoverished country like ours. Until we know the right answer we have to quickly develop a nationwide strategy to prepare for the worse.

According to Phua et al. ${ }^{11}$ as of 2017, Bangladesh had 0.7 critical care bed per 100000 population. And it had 5.23\% population above 65 years. As of 2016 per capita health care expenditure was 34.2 US \$. Health expenditure percentage of GDP was only 2.4. It is quite obvious if Bangladesh has to face a COVID -19 challenge like the one western countries are facing now, our nation will face a miserable situation because of our poor critical care bed capacity and inadequate health care spending.
According to staff report of daily Bonik Barta (bangla language daily newspaper), as of Jan 2019 there were 1074 ICU beds in 70 private hospitals in Bangladesh. There were 221 ICU beds in govt. medical College hospitals, institutions, and district hospitals. The report claims, under normal circumstances there is a need of ten thousand ICU beds equipped with mechanical ventilators among 137000 hospital beds in our country ${ }^{12}$ (not to mention the ventilator requirement we will face if COVID pandemic turns into massive proportion like in Italy or USA).

We also should not forget the fact that because of inadequate number of critical care beds in govt. run hospitals where most of our poor population go for health care, there is an unwritten rationing of ICU beds. Under these circumstances we have to develop policies regarding appropriateness of ICU admissions, cardio pulmonary resuscitation and ventilator candidates ${ }^{13}$.

It was the polio epidemic of 1952, in Copenhagen a little-known event which marked the start of intensive-care medicine and the use of mechanical ventilation. This very care is now at the heart of abating the COVID-19 crisis. In some hospitals, particularly in low-income countries, what is considered an ICU bed is simply one that it is equipped to provide supplemental oxygen to a patient, but not with a ventilator. In the COVID-19 pandemic, the specter of care without ventilators has become common place, even in best provisioned countries of the world ${ }^{14}$.

With latest estimates, at present Bangladesh might have at the most 1400 functioning mechanical ventilators both Govt. and privately owned combined. Out of them 1000 might be commissioned by our ministry of health to be used as ventilators dedicated to care of COVID patients. Because of unusually small number of critically ill COVID patients observed now a days we are yet to face the ultimate crisis of ventilator shortage. At the moment luckily there is no report of acute ventilator shortage at our hospitals with COVID ICUs designated by Bangladesh Govt. But ventilator shortage will be obvious when number of COVID ICUs will grow in near future.

A possible (temporary) solution for the understock of ventilators as observed in Bangladesh is to ventilate multiple patients with one single intensive care ventilator ${ }^{15}$. This is a lucrative concept for us. The modern ventilators have substantial reserves with respect to tidal volume and pressure delivered, which makes it technically possible to ventilate two patients with one single ventilator. This technique should only be used as a temporary bridge until the patient is transported to a location where sufficient ventilators are available, or until new ventilators are available for the patient at the current location. However using one ventilator to serve more than one patient in ICU has not been clinically tested in ICU setting as per literature review. In this scenario the tidal volume (VT) cannot be controlled for each subject and VT disparity is proportional to the variability in compliance ${ }^{16}$. Along with other practical limitations, these findings cannot support the use of this concept for mass-casualty respiratory failure as observed in COVID stricken western countries some of which are suffering from ventilator shortage. 
It is our common sense observation that inadequate number of trained and qualified critical care physicians, critical care nurses and infection control personnel will compound our inability to face the challenge of anticipated COVID -19 pandemic if it turns out to be out of proportion in Bangladesh. Unfortunately we have not yet developed specialized course on critical care nursing in our country. As of March 2020 we have only 35 post graduate MD qualified critical care physicians. In reality under normal circumstances we need at least 600 post graduate critical care physicians. However we can partially overcome the shortage of care givers of COVID patients (in both ICU and non ICU settings) in our district hospitals by tele video conferencing from regional centers staffed with physicians specially trained in COVID management.

In a resource constrained country like Bangladesh, developing an organized supply chain response to the coronavirus outbreak is extremely challenging, given the scale of the crisis and the rate at which it is evolving. Whenever we anticipate the arrival of global crises such as the COVID -19 outbreak, our firms (supplying medicine, PPE, masks and other consumables) can mitigate its impacts by taking supply chain preparedness to a higher level. They should act before a disruption occurs and adjust and execute new plans to ensure uninterrupted supply chain ${ }^{17}$.

There is a welcome news from the World Bank which most recently approved a fast-track $\$ 100$ million financing to help Bangladesh prevent, detect, and respond to the COVID-19 pandemic and strengthen its national systems for public health emergencies ${ }^{18}$.This money will help the COVID-19 Emergency Response and Pandemic Preparedness Project to roll out nation-wide and will upgrade selected health facilities and laboratories to detect, manage and treat suspected and confirmed COVID-19 cases and support screening in Bangladesh's designated health facilities and entry points. It will also benefit people with suspected and confirmed infections, at-risk populations, medical and emergency personnel, as well as public and private service providers, medical and testing facilities, and the national health system.

Over last few months as the pandemic has appeared across the globe with full fury we have seen the rush of management and prevention guidelines published by different international bodies and societies and our ministry of health also has published similar guidelines. In any resource poor setting, in order to protect the vulnerable mass, implementation of any guide line must ensure the best and judicious utilization of its limited resources with special emphasis on preventive measures like social distancing, universal use of face masks, use of soap and hand sanitizer, local quarantining of exposed population, and (as applicable) nationwide or local lockdown etc.

At the moment critical care physicians need to redefine their role and appropriately share the burden of responsibility on the face of this great healthcare challenge of twenty first century. According to one altruism-advocating author, though under normal circumstances, critical care physicians would reject guidelines that did not reflect the highest-quality evidence, in the face of dwindling resources, the workforce seemingly has no choice but to make the most of what is available. As the pandemic spreads around the world, they will continue to be plagued by resource constraints that will compromise their ability to protect themselves, their patients, and their communities but the professional spirit of critical care physicians will march on, unconstrained ${ }^{19}$.

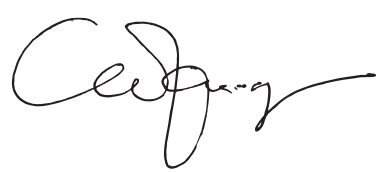

Mohammad Omar Faruq

MD, FACP, FACEP, FCCM, FCPS

Professor of Critical Care Medicine

Chief Consultant, General ICU and Emergency.

United Hospital Ltd. Dhaka 1212, Bangladesh.

President, Bangladesh Society of Critical Care Medicine.

E mail: faruqmo@yahoo.com

\section{References:}

1) Institute of Epidemiology, Disease Control and Research, Dhaka, Bangladesh. www.iedcr.gov.bd

2) Claire Mills. Update on the coronavirus disease COVID-19 pandemic. IN FOCUS. Médecins Sans Frontières' Interview 16 March 2020. www.msf.org

3) Vincent J-L, Taccone F S. Understanding path ways to death in patients with COVID - 19. April 06 2020. DOI: https://doi.org/10.1016/S2213-2600(20)30165-X.D.

4) Ismail $\mathrm{S}$, Baker T, Baker P, Chalkidou K, Chi Y-L and Sullivan R. Strengthening the Basics: Approaches to COVID -19 Care in low resource settings. Center for Global Development .April 82020. www.cgdev.org.

5) Phua J, Weng L, Ling L, Egi M, Lim C-m, Divatia JV et al. Intensive care management of coronavirus disease 2019 (COVID-19): challenges and recommendations. Lancet Respir Med. 2020; (published online April 6. 2020) https://doi.org/ 10.1016/S2213-2600(20)30161-2.

6) Geiling J , Burkle Jr FM , Amundson D , Dominguez-Cherit G , Gomersall CD , Lim ML et al On behalf of the Task Force for Mass Critical Care . Resource-Poor Settings: Infrastructure and Capacity Building Care of the Critically Ill and Injured During Pandemics and Disasters: CHEST Consensus Statement. 146\#4 CHEST OCTOBER 2014 SUPPLEMENT.

7) Wu X, Xu X, Wang X. Six lessons from China's Zhejiang Province and Hangzhou on how countries can prevent and rebound from an epidemic like COVID-19. Image: REUTERS.12 Mar 2020.

8) Atul Gwande. Keeping the corona virus from infecting health care workers. What Singapore's and Hong Kong's success is teaching us about the pandemic? The New Yorker. March 212020.

9) Bangladesh population 2020. www.worldometers.info> bangladesh-population.

10) World Bank national account data and OECD national accounts data files. https://data.worldbank.org/indicator/NY.GDP.MKTP.CD? locations $=\mathrm{BD}$.

11) Phua J, Faruq MO, Kulkarni AP, Redjeki IS, Mendsaikhan N, Sann KK et al. Critical Care Bed Capacity in Asian Countries and Regions. Critical Care Medicine. Jan 2020.DOI: 10.1097/CCM.0000000000004222. 
Bangladesh Crit Care J March 2020; 8 (1): 1-4

12) Govt. hospitals have increased number of beds but ICU care has not improved. Jan 2019. Daily Bonik Barta . www.bonikbarta.com .

13) Faruq MO, Habib SH. Cutting cost without compromising quality can we deliver ICU care to our poor. Bangladesh Crit Care J. Sept $2018 ; 6(2) ; 62-4$

14) Hannah Wunsch. The outbreak that invented Intensive care. Nature. World View. April 03, 2020.doi: 10.1038/d41586-020-01019-y

15) de Vries H, Verdaasdonk R, deJongh F, Vriens M, Doorduin J, Heunks L. Ventilating multiple patients with one ventilator in a crises situation. Dutch Society of Intensive Care. www.NVIC.nl . March 25th, 2020.
16) Branson R, Blakeman T, Robinson B, Johannigman J. Use of a single ventilator to support 4 patients: laboratory evaluation of a limited concept. Respir Care. 2012; 57(3):399-403. doi:10.4187/respcare.01236.

17) James R Rice Jr. Prepare your supply chain for corona virus. Harvard Business Review. Operational management. Feb 272020.

18) World Bank Fast -Tracks $\$ 100$ Million COVID -19 Support for Bangladesh. Press release April 3, 2020. www.worldbank.org .

19) Lisa Rosenbaum. Harnessing Our Humanity - How Washington's Health Care Workers Have Risen to the Pandemic Challenge. NEJM April 1, 2020. DOI: 10.1056/NEJMp2007466. 